\title{
Medizinische Praxiskoordinatorin mit eidgenössischem Fachausweis
}

\author{
Bruno Gutknecht ${ }^{\mathrm{a}}$, Thomas Heuberger \\ a Sekretär OdA MPA; ${ }^{b}$ Dr. med., Präsident OdA Berufsbildung Medizinische Praxisassistentin
}

Anlässlich einer kleinen Prüfungsfeier haben am 15. Dezember 201529 Absolventinnen der ersten Berufsprüfung Medizinische Praxiskoordinatorin / Medizinischer Praxiskoordinator mit eidgenössischem Fachausweis aus der Hand von odamed-Präsident Dr. med. Thomas Heuberger den Ausweis für ihre erfolgreich bestandene Prüfung entgegennehmen können. Dr. med. Emil Schalch gratulierte den Absolventinnen in seiner Ansprache zur Feier herzlich und rief die wichtige Funktion der weitergebildeten Medizinischen Praxisassistentin, nun Praxiskoordinatorin, in der zukünftigen Grundversorgung in der ambulanten Medizin in Erinnerung. Eine spezielle Erwähnung und Ehrung kam Sibylle Has-Grab und Angela Wyss zu, die beide einen Gesamtnotendurchschnitt von 5,9 erzielt haben.

Sie werden in ihrer neuen Funktion entweder chronisch kranke Patienten betreuen oder in grossen Praxisstrukturen teamleitende Funktionen einnehmen.

Die Absolventinnen hatten anlässlich der Prüfung eine schriftliche Fallstudie zu einem vorgegebenen Thema aus den Gebieten "Chronic Care Management» oder "Qualitätsmanagement in der Arztpraxis» vorgelegt. Am Prüfungsanlass vom 3. Dezember 2015 hatten die Absolventinnen ihre Arbeit den Experten zu präsentieren und sich einem Fachgespräch dazu zu stel-

Korrespondenz: Dr. med. Thomas Heuberger OdA Berufsbildung Medizinische Praxisassistentin

Postfach 6432 CH-3001 Bern Tel. 0313805458 Fax 0313810457

\section{Absolventinnen klinischer Fachrichtung}

Nicole Abbühl-Zurbuchen, Muri b. Bern; Renate Beeler, Kerns; Karin Blaser, Grosshöchstetten; Judith Glarner, Wohlen; Miriam Grob, Rothenthurm; Jessica Hubmann, Dieterswil; Karin Kuhn, Dietwil; Sabina Paoletti, Oberglatt; Andrea Rinderknecht, Zürich; Nicole Thönen, Belp; Claudia Wegmann, Wermatswil; Ramona Wespi, Buochs; Christina Sarah Zollinger, Rüti; Sabrina Nicole Zumsteg, Hunzenschwil. len. Den Abschluss des Prüfungsanlasses bildete die EDV-gestützte schriftliche Prüfung.

In den ambulanten Arztpraxen werden jetzt also 14 Praxiskoordinatorinnen klinischer Richtung und 15 Praxiskoordinatorinnen praxisleitender Richtung tätig sein. Sie werden in ihrer neuen Funktion entweder chronisch kranke Patienten beraten und betreuen oder in grossen Praxisstrukturen teamleitende Funktionen einnehmen. In ihrer auf die Prüfung zu führenden Ausbildung haben sie die nötigen Kompetenzen in sechs Modulen mit insgesamt gegen 300 Lektionen Unterricht erworben. Einen zentralen Raum nehmen in beiden Fachrichtungen die vertieften Kenntnisse im Chronic Care Management und im Qualitätsmanagement in der Arztpraxis ein. In der klinischen Richtung werden die Studierenden auf die praktische Beratungstätigkeit am chronisch kranken Patienten vorbereitet. Das Modul «Diabetes» ist Pflichtfach, ergänzend müssen zwei Module aus den Bereichen Atemwegserkrankungen, Koronare Herzkrankheiten/Herzinsuffizienz, Rheuma, Wundversorgung oder Hirnleistungsschwäche absolviert werden. Die Praxiskoordinatorinnen praxisleitender Richtung setzen ihren Schwerpunkt auf Praxismanagement und Personalführung und wahlweise auf Rechnungswesen, erweiterte EDVKenntnisse oder die Wiederaufbereitung von Medizinprodukten. In beiden Fachrichtungen kann das Dosisintensive Röntgen als Wahlfach gewählt werden. Die nächste Berufsprüfung findet am 2. Juni 2016 statt.

\section{Absolventinnen praxisleitender Richtung}

Fabienne Bösch-Zimmermann, Buchs; Bettina Büchel, Heerbrugg; Stephanie Copat, Zürich; Nicole Elsasser, Wetzikon; Cornelia Eugster, Frauenfeld; Corinne Pereira, Rapperswil; Sibylle Has-Grab, Schindellegi; Corina Hitz, Chur; Caroline Lehner, Dinhard; Nicole Lopez Cuyun-Meier, Luzern; Rebecca Rüegg, Goldingen; Nicole Sanin, Tann; Nadine von Euw, Greifensee; Angela Wyss, Winterthur; Aline Zünd, Olten. 